\title{
Ultrafast Spectroelectrochemistry Reveals Photoinduced Carrier Dynamics in Positively Charged CdSe Nanocrystals
}

Honarfar, Alireza; Chabera, Pavel; Lin, Weihua; Meng, Jie; Mourad, Hassan; Pankratova, Galina; Gorton, Lo; Zheng, Kaibo; Pullerits, Tõnu

Published in:

Journal of Physical Chemistry C

Link to article, DOI:

10.1021/acs.jpcc.1c02729

Publication date:

2021

Document Version

Publisher's PDF, also known as Version of record

Link back to DTU Orbit

Citation (APA):

Honarfar, A., Chabera, P., Lin, W., Meng, J., Mourad, H., Pankratova, G., Gorton, L., Zheng, K., \& Pullerits, T. (2021). Ultrafast Spectroelectrochemistry Reveals Photoinduced Carrier Dynamics in Positively Charged CdSe Nanocrystals. Journal of Physical Chemistry C, 125(26), 14332-14337. https://doi.org/10.1021/acs.jpcc.1c02729

\section{General rights}

Copyright and moral rights for the publications made accessible in the public portal are retained by the authors and/or other copyright owners and it is a condition of accessing publications that users recognise and abide by the legal requirements associated with these rights.

- Users may download and print one copy of any publication from the public portal for the purpose of private study or research.

- You may not further distribute the material or use it for any profit-making activity or commercial gain

- You may freely distribute the URL identifying the publication in the public portal 


\title{
Ultrafast Spectroelectrochemistry Reveals Photoinduced Carrier Dynamics in Positively Charged CdSe Nanocrystals
}

\author{
Alireza Honarfar, Pavel Chabera, Weihua Lin, Jie Meng, Hassan Mourad, Galina Pankratova, Lo Gorton, \\ Kaibo Zheng,* and Tõnu Pullerits*
}

Cite This: J. Phys. Chem. C 2021, 125, 14332-14337

Read Online

ACCESS

Wll Metrics \& More

Article Recommendations

Supporting Information

ABSTRACT: Extra charges in semiconductor nanocrystals are of paramount importance for their electrically driven optoelectronic and photovoltaic applications. Optical excitations of such charged nanocrystals lead to rapid recombination via an Auger process, which can deteriorate the performance of the corresponding devices. While numerous articles report trion Auger processes in negatively charged nanocrystals, optical studies of well-controlled positive charging of nanocrystals and detailed studies of positive trions remain rare. In this work, we used electrochemistry to achieve positive charging of CdSe nanocrystals, so-called quantum dots (QDs), in a controlled way. Femtosecond transient absorption spectroscopy was applied for in situ investigation of the charge carrier dynamics after optical excitation of the electrochemically charged QD assembly on

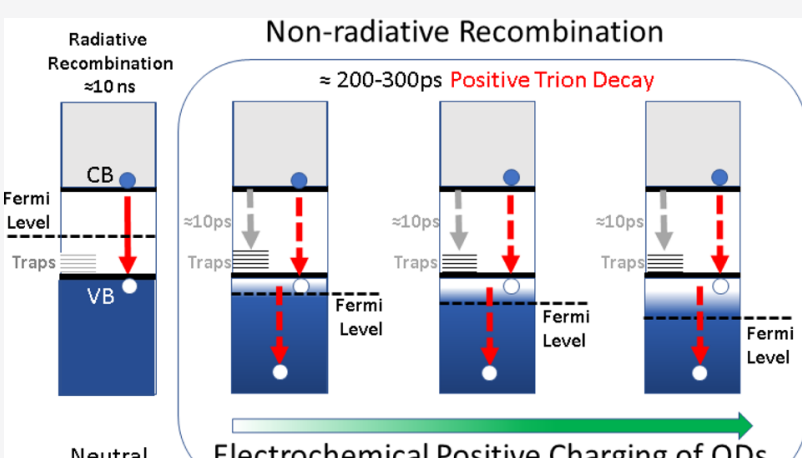
$\mathrm{TiO}_{2}$. We observe that without bias (i.e., neutral QDs), subpicosecond hot carrier cooling is followed by multiple phases of the dynamics corresponding to electron injection and transfer to the $\mathrm{TiO}_{2}$. Positive charging first leads to activation of the hole traps close to the valence band maximum, which opens a rapid recombination channel of the optical excitation. A further increase in the positive bias interrupts the electron injection to $\mathrm{TiO}_{2}$, and if nanocrystals are positively charged, it leads to Auger relaxation in a few hundred picosecond timescale. This study represents a step toward the understanding of the effect of positive charging on the performance of semiconductor nanocrystals under conditions which closely mimic their potential applications.

\section{INTRODUCTION}

At the nanoscale, material properties depend on their size owing to the quantum confinement. For example, absorption and emission of semiconductor nanocrystals with a size smaller than the exciton Bohr radius, the so-called quantum dots (QDs), ${ }^{1,2}$ can be tuned by changing their size. Since colloidal QDs can be made by facile wet-chemistry methods, ${ }^{3}$ they have become popular model systems for basic research ${ }^{4-8}$ and are already used in photodriven applications as fluorophores in high-color purity displays. ${ }^{1,9}$ Recently, an electrically driven optical gain in QDs was reported, demonstrating the potential of such nanomaterials for optoelectronics and realization of QD lasing. ${ }^{10,11}$

When a QD absorbs a photon with energy larger than its band gap, an electron-hole pair is created, which is referred to as an exciton. ${ }^{12}$ Biexciton is a pair of excitons, which can rapidly decay via a nonradiative Auger mechanism. ${ }^{13}$ In this process, one exciton's recombination energy is transferred to the electron or hole of the other exciton, bringing one of the charge carriers to a high energy level, which then rapidly relaxes back to the band edge. ${ }^{14}$ As a result of such a process, one exciton is lost. If a $\mathrm{QD}$ is charged (negatively or positively), light absorption leads to the formation of a socalled trion-a collective three-body state consisting of an exciton combined with an additional charge: a hole or an electron. ${ }^{13}$ In trions too, an Auger process takes place analogously to the biexcitons. ${ }^{13}$ In optoelectronic applications, QDs are often highly excited or charged; therefore, the Auger recombination is an important mechanism, which can significantly affect the efficiency of the devices. Auger recombination can be either detrimental ${ }^{15}$ or can be beneficial for the performance of the device. ${ }^{16}$ Clearly, for making the best-possible use of the nanomaterials, photophysics of charged QDs needs to be better understood.

Photocharging is probably the most straightforward way to make charged QDs for spectroscopic studies. ${ }^{17,18}$ The charging is typically confirmed by shortening of the QD photo-

Received: March 26, 2021

Revised: June 10, 2021

Published: June 24, 2021 
luminescent lifetime due to the trion Auger decay. ${ }^{13}$ However, it is not a priori known whether a positive or negative trion has been formed by the photocharging. For a controlled charging of QDs, photochemical doping ${ }^{19-21}$ or electrochemistry methods ${ }^{15,22-25}$ can be used. While the negative trion properties have been extensively studied, ${ }^{13,15,20,26}$ only a few articles address the photophysics of the positively charged QDs. ${ }^{20,21,27}$ Partially, this is related to the fact that in QDs, the spectroscopic signatures of valence band (VB) holes are much weaker than the state-filling signal from the conduction band electrons. $^{28,29}$ Earlier, the hole-related trions have been studied using photochemical doping to prepare positively charged $\mathrm{CdSe} / \mathrm{CdS}$ core shell QDs. ${ }^{20,21,27}$

In the following, we use spectroelectrochemistry as a direct control method for positive charging of the QDs in combination with in situ spectroscopic studies. We measure the $I-V$ charging curve using cycling voltammetry while simultaneously following the QD steady-state absorption changes in order to quantify the charging effect. We also applied ultrafast transient absorption (TA) spectroscopy at certain positive potentials to study the photoexcitation dynamics of positively charged QDs. Our results demonstrate the potential of electrochemistry as a versatile tool that can be used for charging of QDs in a well-controlled way. Electrochemistry can be used in combination with other techniques such as spectroscopy for in-depth studies of the effect of excess charges on the device performance.

\section{METHODS}

QD Synthesis. For the synthesis of the CdSe nanoparticles, a conventional hot injection method was used. ${ }^{3,30}$ A total of $1500 \mathrm{mg}$ of $\mathrm{CdO}$ was dissolved in $7 \mathrm{~mL}$ of OLEA and $50 \mathrm{~mL}$ of ODE at $270{ }^{\circ} \mathrm{C}$ in a three-neck flask. A Se precursor was prepared by sonication of $300 \mathrm{mg}$ of Se powder in $10 \mathrm{~mL}$ of ODE for $10 \mathrm{~min}$ in an Ar-purged flask; then, $1 \mathrm{~mL}$ of trioctylphosphine was added and stirred until the solution became completely transparent. The temperature of the $\mathrm{Cd}$ precursor solution was lowered to $240{ }^{\circ} \mathrm{C}$; then, the Se solution was quickly injected. When the QDs of the desired size were obtained, the flask was removed from the heater and quickly cooled with an ice bath. All chemicals were purchased from Sigma-Aldrich and used without any further purification or modification.

Film Preparation. The fluorine-doped tin oxide (FTO) slides were coated using the doctor blade technique with $\mathrm{TiO}_{2}$ paste purchased from Solaronix, sintered at $480{ }^{\circ} \mathrm{C}$, and cooled overnight. A $\mathrm{TiO}_{2}$ mesoporous layer was used for $\mathrm{QD}$ anchoring and to achieve a large surface area, thereby providing sufficient optical density for spectroscopy measurements. The capping ligand was changed to 3-mercaptopropionic acid. Films were prepared by soaking $\mathrm{TiO}_{2}$-coated FTO slides in aqueous solution of QDs and were adjusted to $\mathrm{pH}=$ 12 by dropwise addition of $\mathrm{NaOH}$. Films were then washed with distilled water and heated to $100{ }^{\circ} \mathrm{C}$. Films were stored in an Ar-filled desiccator over drying agents in the dark.

Electrochemistry. A conventional three-electrode electrochemical system in a custom-made spectroelectrochemical cell was used. ${ }^{31}$ QD-sensitized $\mathrm{TiO}_{2}$-coated FTO was used as the working electrode, a $\mathrm{Pt}$ wire as the counter electrode, and a leak-less $\mathrm{Ag} \mid \mathrm{AgCl}$ was used as the pseudo-reference electrode. The electrolyte solution was a $0.1 \mathrm{M}$ solution of tetrabutylammonium hexafluorophosphate in dichloromethane (DCM). A scan rate of $1 \mathrm{mV} / \mathrm{s}$ was used for running cyclic voltammogram $(\mathrm{CV})$; it ensures a quasi-stationary condition for the measurements. A PalmSense 4 potentiostat was used for all electrochemical measurements. The corresponding stationary open-circuit potential (OCP) was $-0.25 \mathrm{~V}$ versus AglAgCl.

Spectroelectrochemistry. Measurements were done in the same electrochemical cell as mentioned above. UV-vis steady-state absorption spectra were recorded using an Agilent spectrophotometer during the electrochemical cyclic voltammetry scan. Spectral measurements were done at a limited number of specific potentials to prevent photocharging or photocurrents from distorting the peaks in $\mathrm{CV}$, which can further complicate the interpretation. The acquisition of each spectrum took about $5 \mathrm{~s}$, causing a negligible effect on the current and during which the potential changes only by \pm 5 $\mathrm{mV}$.

TA Spectroscopy. Measurements were done in the same electrochemical cell as mentioned earlier. Output pulses of a Spitfire Pro regenerative amplifier $(796 \mathrm{~nm}, 6 \mathrm{~mJ}, 100 \mathrm{fs})$ were used to generate both pump and probe light. The second harmonic of the fundamental laser wavelength generated by a BBO crystal was used as pump pulses with a central wavelength of $398 \mathrm{~nm}$. To avoid the multiexcitations, a very low photon flux was used (50 $\mu \mathrm{W}$ with $400 \mu \mathrm{m}$ spot size). Output of a NOPA (TOPAS) was used to generate $1300 \mathrm{~nm}$ pulses, which were focused in a $\mathrm{CaF}_{2}$ crystal to generate broadband white light ( 350 to $1200 \mathrm{~nm}$ ) to be used as the probe. For TA, we used bulk electrolysis or chronoamperometric methods. After setting a specific potential and reaching equilibrium (i.e., steady current with no changes), spectral evolution of the photoexcited QDs was recorded at different delay times between the laser pump and probe. The data are collected as average of 1000 laser pulses at each delay point. In our measurement, the negative signal corresponds to the ground-state bleach together with stimulated emission, and the positive signal is the excited-state absorption. Stability of the measurement was checked by comparing the amplitude of the TA signal at each scan. For each measurement, at least, five full scans are measured and averaged.

TA Data Analysis. To decipher the TA measurements under different potentials, global fitting was used as the main data analysis method. Glotaran software was used for the analyses. For visualizing the results, Origin software was used.

\section{RESULTS AND DISCUSSION}

For estimation of the band structure of the CdSe QDs, we used cyclic voltammetry as a common tool for measuring the energy level positions in semiconductors. ${ }^{15,23,32}$ Measurement of CV of the QDs was done in a three-electrode electrochemical cell. The QD assembly onto $\mathrm{TiO}_{2}$-FTO serves as the working electrode, and a platinum plate is used as the counter electrode (see the Methods section). The potentials are measured and reported versus a $\mathrm{Ag} \mid \mathrm{AgCl}$ pseudo-reference electrode. A representative $\mathrm{CV}$ is shown in Figure 1.

The peaks in the $\mathrm{CV}$ represent significant changes in the current and correspond to the potentials where electron exchange is taking place between the working electrode (i.e., $\mathrm{TiO}_{2}$-FTO) and the sample (in this case CdSe QDs) in the electrochemical cell. In our measurements, it means positive charging of the QDs. Since in the electrochemical measurements, the potential is regulated versus a reference electrode with a relatively fixed potential, the change toward the positive potentials corresponds to the lowering of the Fermi level of 


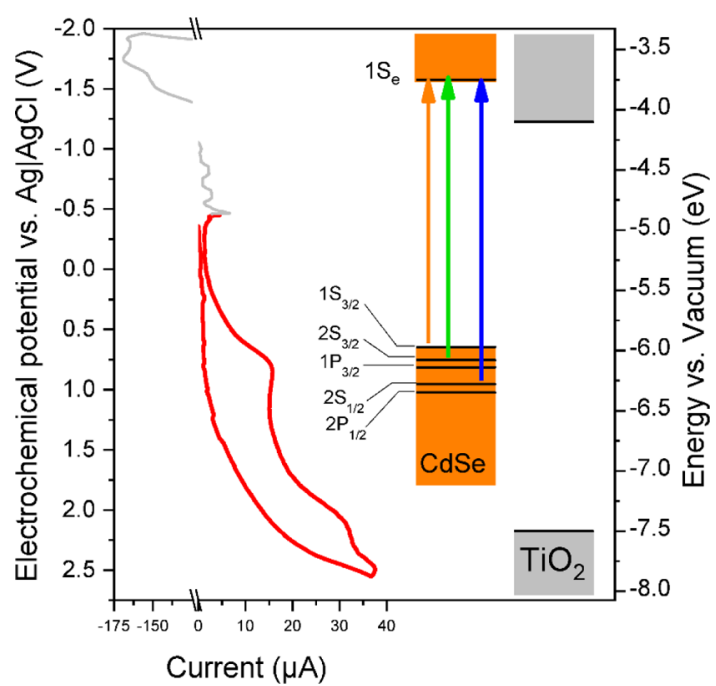

Figure 1. Correlation between electrochemical measurement and the band structure of the $3 \mathrm{~nm} \mathrm{CdSe} \mathrm{QDs}$ and $\mathrm{TiO}_{2}$. Electrochemical measurement was performed in a three-electrode cell, where the CdSe $\mathrm{QD}$ assembly on mesoporous $\mathrm{TiO}_{2}$-coated FTO serves as the working electrode (FTO is not shown). A leak-less $\mathrm{Ag} / \mathrm{AgCl}$ electrode was used as the reference, and a platinum plate was used as the counter electrode (supporting electrolyte: $0.1 \mathrm{M}$ tetra butyl ammonium hexafluorophosphate in DCM, scan rate of $1 \mathrm{mV} / \mathrm{s}$, room temperature). The peaks at -1.75 and $+0.75 \mathrm{~V}$ are assigned to the conduction band (first excited state) and valance band of $3 \mathrm{~nm} \mathrm{CdSe}$ QDs, respectively. The band structure of $3 \mathrm{~nm}$ CdSe QDs is shown, where the arrows indicate optically allowed transitions.

$\mathrm{TiO}_{2}$-FTO. Such conjecture is based on the fact that $\mathrm{TiO}_{2}-$ FTO slides are in direct contact with each other and the Fermi level should be equal throughout their junction. We can assume the QD assembly as another semiconductor, which is separated by a thin insulator (ligand) and in this way correlates the electrochemical potential to the QD energy levels. In the $\mathrm{CV}$ shown in Figure 1, we can clearly see the presence of a peak as the consequence of scanning the applied electrochemical potential toward positive potentials. At around +0.75 $\mathrm{V}$, it is expected that the VB levels become emptied of electrons, leading to a peak in the $\mathrm{CV}$ and accordingly to positive charging of QDs. The optical properties and electronic structure of the QDs will change due to the positive charging ${ }^{33}$ as the Fermi level reaches the band edge of the QDs. ${ }^{23}$ Hence, the properties of the material are alternated and controlled by adjusting the applied electrochemical potential. The changes in the steady-state absorption of QDs due to the spectroelectrochemical charging under positive bias are illustrated in Figure 2. As a comparison, we also show the unbiased QD absorption spectrum, which is measured at OCP. In our measurements, the changes due to the positive charging remain within $10 \%$ of the absorption of neutral QDs. This is qualitatively consistent with the known weak hole-related signal in the QD absorption spectrum, which is partially related to the high density of hole states at the band edge. ${ }^{28,29}$

As the next step, we apply femtosecond TA spectroscopy to in situ probe the excited-state dynamics of the QDs under applied positive bias. The measurements are employed to follow the population and depopulation pathways of the photoexcited positively charged QDs. TA data and the globally fitted exponential decay-associated spectral (DAS) components, obtained through singular value decomposition (SVD),

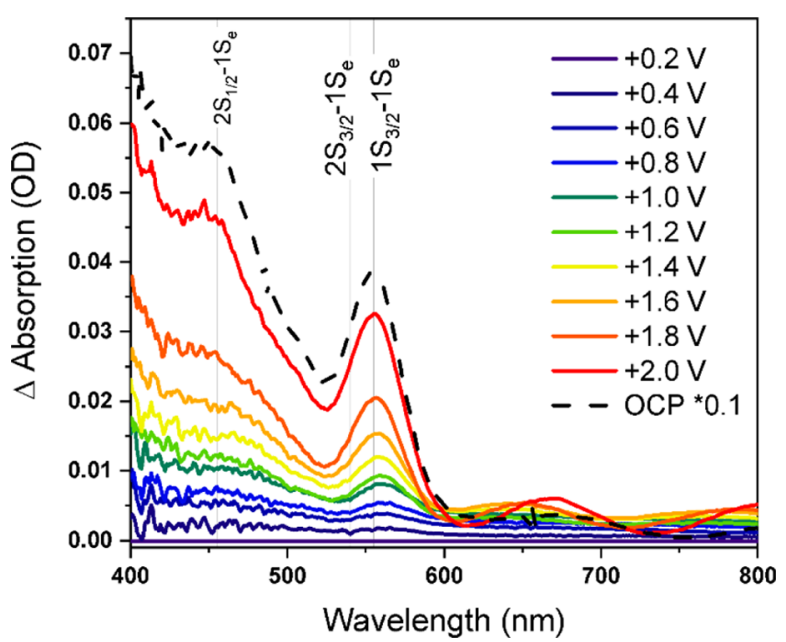

Figure 2. Spectroelectrochemical measurements of $3 \mathrm{~nm} \mathrm{CdSe} \mathrm{QDs}$ on $\mathrm{TiO}_{2}$-FTO. ( $\mathrm{OCP}=-0.25 \mathrm{~V}$ vs $\left.\mathrm{Ag} / \mathrm{AgCl}\right)$. Absorption spectra were recorded during the electrochemical measurement. Potentialdependent difference spectra are constructed by subtraction of the OCP spectrum from the measured spectra. The OCP spectrum is scaled by 0.1 for better comparison.

are plotted in Figure 3. In order to distinguish the potentialinduced changes in the QD excited-state dynamics, we use TA measurement at OCP as the reference.

At OCP, the fast component of 400-500 fs lifetime corresponds to the hot electron cooling after excitation with $398 \mathrm{~nm}$ pulses. The component has a characteristic sigmoidal shape, which means a decay of photogenerated charge carriers from higher energies and a rise at the band edge, which is consistent with the carrier relaxation process. ${ }^{28,29,34,35}$ The SVD analyses also find two decay components that are assigned to the electron injection and transfer from the QD conduction band to $\mathrm{TiO}_{2}$. The faster of the two, $\sim 60 \mathrm{ps}$, corresponds to electron injection, while the slower component of several hundred picoseconds is the migration of the injected electron further away from the $\mathrm{QD}-\mathrm{TiO}_{2}$ interface to the bulk of $\mathrm{TiO}_{2}$, thereby losing the interaction with the photogenerated hole, which remains in the QD. ${ }^{36}$ The weak broad positive signal spanning over the near-infrared range (650$1100 \mathrm{~nm}$ ) is due to the absorption of the free charges in the conduction band of $\mathrm{TiO}_{2}{ }^{37-39}$ Eventually, the absorption bleach is recovered by charges recombining with the lifetime, which is longer than our experimental window noted as $>2 \mathrm{~ns}$.

Application of positive potentials leads to changes in the photogenerated charge carrier dynamics. At $+0.5 \mathrm{~V}$, the charge injection to $\mathrm{TiO}_{2}$ and the ground-state bleach recovery become drastically faster. A new component of a few ps appears in the kinetics at $+0.5 \mathrm{~V}$. We interpret the component as the result of the Fermi level reaching surface defect states within the QD band gap close to the VB. These trap states will be emptied (populated by holes). The existence of such states in the coreonly QDs is supported by several studies. ${ }^{29,33,40}$ The states act as hole traps and recombination centers, leading to a ps component of the TA decay. We suggest that the ps component is a combination of the fast sub-picosecond cooling and the ps trapping, which as the result of uncertainty in the global fitting has merged into a single component of 1.6 ps. The generally faster bleach recovery can be partially explained in terms of an increased Coulombic interaction between the now positively charged QDs and the electrons 




Figure 3. Excited-state dynamics of $3 \mathrm{~nm}$ CdSe QDs under applied positive bias. Summary of the TA measurements and their corresponding DAS for $3 \mathrm{~nm}$ CdSe QD assembled on the $\mathrm{TiO}_{2}$-FTO electrode at OCP, $+0.5,+0.75,+1.0$, and $+1.5 \mathrm{~V} v s \mathrm{AglAgCl}$. Cartoon depiction of photogenerated electron transitions at the given potentials. Black arrows indicate laser excitation connecting the corresponding energy levels for photogenerated charges, and blue (down) arrows indicate the decaying pathways and recombination mechanisms.

injected to the $\mathrm{TiO}_{2}$. Since the electron injection-related component of $60 \mathrm{ps}$ is still present, while the slower diffusion phase is not distinguishable from the bleach recovery, we can conclude that electrons remain near the QDs and can recombine more efficiently. The faster recovery may also be the result of intraband states in $\mathrm{TiO}_{2}$, which are activated (changed) by the applied positive potential. Such states in $\mathrm{TiO}_{2}$ are known to be formed by dangling bonds at the surface and oxygen vacancies in the material. ${ }^{38,39,41}$

At $+0.75 \mathrm{~V}$, the Fermi level is shifted to lower energy levels than those of the VB of the QDS. The spectroelectrochemical measurements in Figure 2 clearly show a change in the steadystate absorption of the QDs compared to the lower potentials. Since the density of states at the VB is large and the energy levels are closely packed, we cannot quantify the precise number of holes in the VB per QD. However, we can qualitatively conclude that holes have been injected into the VB of QDs due to the applied electrochemical bias. Upon photoexcitation of such positively charged QDs, a positive trion or positively charged exciton is created. The cooling of hot electrons and the trapping remain unchanged within the experimental and global fitting uncertainty. An important difference compared to the lower potentials apparent from data analysis is that no electron injection to $\mathrm{TiO}_{2}$ can be seen. Also, no broadband excited-state absorption of the free electrons in $\mathrm{TiO}_{2}$ can be detected. We conclude that charge injection to $\mathrm{TiO}_{2}$ is completely terminated. The longest component of the bleach recovery is in the range of $200-300$ ps, which is due to the Auger decay of the positive trion. Further rise in the electrochemical potential to +1.0 and $+1.5 \mathrm{~V}$ does not induce any significant change in the dynamics. Only the hot carrier cooling becomes slightly faster, consistent with the expected more efficient carrier-carrier scattering in the case of higher charging.

\section{CONCLUSIONS}

We have prepared high-optical quality QD-sensitized $\mathrm{TiO}_{2}$ electrodes for combining electrochemical measurements with in situ ultrafast laser spectroscopy. Electrochemical charging is used as a direct manipulation tool to inject and control extra holes in the VB of the QDs. Based on the cyclic voltammogram and changes in the steady-state absorption spectra, we could identify the electrochemical potentials for positive charging of the QDs. Femtosecond TA spectroscopy allowed us to follow the details of the optically excited charge carrier dynamics in such a well-controlled charged system. Besides a subpicosecond hot electron cooling, a fast decay of excitation with a 250 ps time constant due to Auger decay is clearly observable in the TA measurements. Higher potentials which may cause multiple charging did not make any significant difference in the positively charged QD Auger decay lifetime. These observations provide basic information about the positive trion and the potential of spectroelectrochemical measurements for further detailed studies of such processes in QDs. These findings may pave the way for refining the design principles of QD optoelectronics.

\section{ASSOCIATED CONTENT}

\section{SI Supporting Information}

The Supporting Information is available free of charge at https://pubs.acs.org/doi/10.1021/acs.jpcc.1c02729.

Wavelength-integrated TA kinetics at different applied potentials together with the global fit obtained via SVD analyses with Glotaran software (PDF) 


\section{AUTHOR INFORMATION}

\section{Corresponding Authors}

Kaibo Zheng - Chemical Physics and NanoLund, Department of Chemistry, Lund University, 22100 Lund, Sweden; Department of Chemistry, Technical University of Denmark, 2800 Kongens Lyngby, Denmark; 이이.org/0000-00027236-1070; Phone: +4646 22281310;

Email: Tonu.Pullerits@chemphys.lu.se; Fax: +46 46 2224119

Tõnu Pullerits - Chemical Physics and NanoLund, Department of Chemistry, Lund University, 22100 Lund, Sweden; 10 orcid.org/0000-0003-1428-5564;

Email: Kaibo.Zheng@chemphys.lu.se

\section{Authors}

Alireza Honarfar - Chemical Physics and NanoLund, Department of Chemistry, Lund University, 22100 Lund, Sweden; 10 orcid.org/0000-0002-1852-3059

Pavel Chabera - Chemical Physics and NanoLund, Department of Chemistry, Lund University, 22100 Lund, Sweden; 이잉.org/0000-0002-0531-5138

Weihua Lin - Chemical Physics and NanoLund, Department of Chemistry, Lund University, 22100 Lund, Sweden

Jie Meng - Department of Chemistry, Technical University of Denmark, 2800 Kongens Lyngby, Denmark; 이이. orcid/ 0000-0002-3813-5221

Hassan Mourad - Chemical Physics and NanoLund, Department of Chemistry, Lund University, 22100 Lund, Sweden

Galina Pankratova - DTU Nanolab, National Centre for Nano Fabrication and Characterization, Technical University of Denmark, 2800 Kongens Lyngby, Denmark; Department of Biochemistry and Structural Biology, Lund University, 22100 Lund, Sweden

Lo Gorton - Department of Biochemistry and Structural Biology, Lund University, 22100 Lund, Sweden;

() orcid.org/0000-0002-7278-0478

Complete contact information is available at:

https://pubs.acs.org/10.1021/acs.jpcc.1c02729

\section{Author Contributions}

The manuscript was written through contributions of all authors. All authors have given approval to the final version of the manuscript.

\section{Notes}

The authors declare no competing financial interest.

\section{ACKNOWLEDGMENTS}

Authors acknowledge funding by the Swedish Research Council, Swedish Energy Agency, Crafoord Foundation, and NanoLund. A.H. acknowledges the support and discussions from Prof. Ebbe Nordlander, Dr. Alexey Polukeev, and Prof. Ola F. Wendt and Dr. Carlito S. Ponseca, Dr. Ahibur Rahaman, and Dr. Mohamed Abdellah.

\section{REFERENCES}

(1) Colloidal Quantum Dot Optoelectronics and Photovoltaics; Konstantatos, G., Sargent, E. H.; Cambridge University Press: Cambridge, U.K., 2013.

(2) Nanocrystal Quantum Dots, 2nd ed.; Klimov, V. I.; CRC Press, 2017.

(3) Murray, C. B.; Norris, D. J.; Bawendi, M. G. Synthesis and Characterization of Nearly Monodisperse Cde $(\mathrm{E}=$ Sulfur, Selenium,
Tellurium) Semiconductor Nanocrystallites. J. Am. Chem. Soc. 1993, $115,8706-8715$.

(4) Tvrdy, K.; Frantsuzov, P. A.; Kamat, P. V. Photoinduced Electron Transfer from Semiconductor Quantum Dots to Metal Oxide Nanoparticles. Proc. Natl. Acad. Sci. U.S.A. 2011, 108, 29-34.

(5) Shirasaki, Y.; Supran, G. J.; Bawendi, M. G.; Bulović, V. Emergence of Colloidal Quantum-Dot Light-Emitting Technologies. Nat. Photonics 2013, 7, 13-23.

(6) Norris, D. J.; Bawendi, M. G. Measurement and Assignment of the Size-Dependent Optical Spectrum in Cdse Quantum Dots. Phys. Rev. B: Condens. Matter Mater. Phys. 1996, 53, 16338-16346.

(7) Brus, L. E. Electron-electron and electron-hole interactions in small semiconductor crystallites: The size dependence of the lowest excited electronic state. J. Chem. Phys. 1984, 80, 4403-4409.

(8) Alivisatos, A. P. Perspectives on the Physical Chemistry of Semiconductor Nanocrystals. J. Phys. Chem. 1996, 100, 1322613239.

(9) Chen, O.; Wei, H.; Maurice, A.; Bawendi, M.; Reiss, P. Pure colors from core-shell quantum dots. MRS Bull. 2013, 38, 696-702.

(10) Lim, J.; Park, Y.-S.; Klimov, V. I. Optical Gain in Colloidal Quantum Dots Achieved with Direct-Current Electrical Pumping. Nat. Mater. 2018, 17, 42-49.

(11) Geuchies, J. J.; Brynjarsson, B.; Grimaldi, G.; Gudjonsdottir, S.; Van Der Stam, W.; Evers, W. H.; Houtepen, A. J. Quantitative Electrochemical Control over Optical Gain in Quantum-Dot Solids. ACS Nano 2021, 15, 377-386.

(12) Rabouw, F. T.; De Mello Donega, C. Excited-State Dynamics in Colloidal Semiconductor Nanocrystals. Top. Curr. Chem. 2016, 374, 58.

(13) Jha, P. P.; Guyot-Sionnest, P. Trion Decay in Colloidal Quantum Dots. ACS Nano 2009, 3, 1011-1015.

(14) Efros, A. L.; Rosen, M.; Kuno, M.; Nirmal, M.; Norris, D. J.; Bawendi, M. Band-Edge Exciton in Quantum Dots of Semiconductors with a Degenerate Valence Band: Dark and Bright Exciton States. Phys. Rev. B: Condens. Matter Mater. Phys. 1996, 54, 4843-4856.

(15) Honarfar, A.; et al. Photoexcitation Dynamics in Electrochemically Charged CdSe Quantum Dots: From Hot Carrier Cooling to Auger Recombination of Negative Trions. ACS Appl. Energy Mater. 2020, 3, 12525-12531.

(16) Singh, R.; Liu, W.; Lim, J.; Robel, I.; Klimov, V. I. Hot-Electron Dynamics in Quantum Dots Manipulated by Spin-Exchange Auger Interactions. Nat. Nanotechnol. 2019, 14, 1035-1041.

(17) McGuire, J. A.; Sykora, M.; Robel, I.; Padilha, L. A.; Joo, J.; Pietryga, J. M.; Klimov, V. I. Spectroscopic Signatures of Photocharging Due to Hot-Carrier Transfer in Solutions of Semiconductor Nanocrystals under Low-Intensity Ultraviolet Excitation. ACS Nano 2010, 4, 6087-6097.

(18) Makarov, N. S.; Guo, S.; Isaienko, O.; Liu, W.; Robel, I.; Klimov, V. I. Spectral and Dynamical Properties of Single Excitons, Biexcitons, and Trions in Cesium-Lead-Halide Perovskite Quantum Dots. Nano Lett. 2016, 16, 2349-2362.

(19) Rinehart, J. D.; Schimpf, A. M.; Weaver, A. L.; Cohn, A. W.; Gamelin, D. R. Photochemical Electronic Doping of Colloidal Cdse Nanocrystals. J. Am. Chem. Soc. 2013, 135, 18782-18785.

(20) Hou, X.; Kang, J.; Qin, H.; Chen, X.; Ma, J.; Zhou, J.; Chen, L.; Wang, L.; Wang, L.-W.; Peng, X. Engineering Auger Recombination in Colloidal Quantum Dots Via Dielectric Screening. Nat. Commun. 2019, 10, 1750.

(21) Wu, K.; Lim, J.; Klimov, V. I. Superposition Principle in Auger Recombination of Charged and Neutral Multicarrier States in Semiconductor Quantum Dots. ACS Nano 2017, 11, 8437-8447.

(22) Houtepen, A. J.; Vanmaekelbergh, D. Orbital Occupation in Electron-Charged Cdse Quantum-Dot Solids. J. Phys. Chem. B 2005, 109, 19634-19642.

(23) Spittel, D.; Poppe, J.; Meerbach, C.; Ziegler, C.; Hickey, S. G.; Eychmüller, A. Absolute Energy Level Positions in Cdse Nanostructures from Potential-Modulated Absorption Spectroscopy (Emas). ACS Nano 2017, 11, 12174-12184. 
(24) Boehme, S. C.; Walvis, T. A.; Infante, I.; Grozema, F. C.; Vanmaekelbergh, D.; Siebbeles, L. D. A.; Houtepen, A. J. Electrochemical Control over Photoinduced Electron Transfer and Trapping in Cdse-Cdte Quantum-Dot Solids. ACS Nano 2014, 8, 7067-7077.

(25) Wang, C.; Wehrenberg, B. L.; Woo, C. Y.; Guyot-Sionnest, P. Light Emission and Amplification in Charged Cdse Quantum Dots. J. Phys. Chem. B 2004, 108, 9027-9031.

(26) Galland, C.; Ghosh, Y.; Steinbrück, A.; Hollingsworth, J. A.; Htoon, H.; Klimov, V. I. Lifetime Blinking in Nonblinking Nanocrystal Quantum Dots. Nat. Commun. 2012, 3, 908.

(27) Park, Y.-S.; Bae, W. K.; Pietryga, J. M.; Klimov, V. I. Auger Recombination of Biexcitons and Negative and Positive Trions in Individual Quantum Dots. ACS Nano 2014, 8, 7288-7296.

(28) Zheng, K.; Žídek, K.; Abdellah, M.; Zhang, W.; Chábera, P.; Lenngren, N.; Yartsev, A.; Pullerits, T. Ultrafast Charge Transfer from Cdse Quantum Dots to P-Type Nio: Hole Injection Vs Hole Trapping. J. Phys. Chem. C 2014, 118, 18462-18471.

(29) Grimaldi, G.; Geuchies, J. J.; Van Der Stam, W.; du Fossé, I.; Brynjarsson, B.; Kirkwood, N.; Kinge, S.; Siebbeles, L. D. A.; Houtepen, A. J. Spectroscopic Evidence for the Contribution of Holes to the Bleach of Cd-Chalcogenide Quantum Dots. Nano Lett. 2019, 19, 3002-3010.

(30) Peng, X.; Manna, L.; Yang, W.; Wickham, J.; Scher, E.; Kadavanich, A.; Alivisatos, A. P. Shape Control of Cdse Nanocrystals. Nature 2000, 404, 59-61.

(31) Honarfar, A. Charge Carrier Dynamics in Novel Solar Materials : Ultrafast Spectroelectrochemistry. Ph.D. Dissertation, Lund University, Lund, 2021.

(32) Ingole, P. P. A Consolidated Account of Electrochemical Determination of Band Structure Parameters in Ii-Vi Semiconductor Quantum Dots: A Tutorial Review. Phys. Chem. Chem. Phys. 2019, 21, $4695-4716$.

(33) du Fossé, I.; Ten Brinck, S.; Infante, I.; Houtepen, A. J. Role of Surface Reduction in the Formation of Traps in n-Doped II-VI Semiconductor Nanocrystals: How to Charge without Reducing the Surface. Chem. Mater. 2019, 31, 4575-4583.

(34) Zídek, K.; Zheng, K.; Abdellah, M.; Lenngren, N.; Chábera, P.; Pullerits, T. Ultrafast Dynamics of Multiple Exciton Harvesting in the Cdse-Zno System: Electron Injection Versus Auger Recombination. Nano Lett. 2012, 12, 6393-6399.

(35) Zheng, K.; Karki, K.; Žídek, K.; Pullerits, T. Ultrafast Photoinduced Dynamics in Quantum Dot-Based Systems for Light Harvesting. Nano Res. 2015, 8, 2125-2142.

(36) Žídek, K.; Zheng, K.; Ponseca, C. S.; Messing, M. E.; Wallenberg, L. R.; Chábera, P.; Abdellah, M.; Sundström, V.; Pullerits, T. Electron Transfer in Quantum-Dot-Sensitized Zno Nanowires: Ultrafast Time-Resolved Absorption and Terahertz Study. J. Am. Chem. Soc. 2012, 134, 12110-7.

(37) Maity, P.; Mohammed, O. F.; Katsiev, K.; Idriss, H. Study of the Bulk Charge Carrier Dynamics in Anatase and Rutile $\mathrm{TiO}_{2}$ Single Crystals by Femtosecond Time-Resolved Spectroscopy. J. Phys. Chem. C 2018, 122, 8925-8932.

(38) Rossi, G.; Pasquini, L.; Catone, D.; Piccioni, A.; Patelli, N.; Paladini, A.; Molinari, A.; Caramori, S.; O’Keeffe, P.; Boscherini, F. Charge Carrier Dynamics and Visible Light Photocatalysis in Vanadium-Doped $\mathrm{TiO}_{2}$ Nanoparticles. Appl. Catal., B 2018, 237, 603-612.

(39) Schneider, J.; Matsuoka, M.; Takeuchi, M.; Zhang, J.; Horiuchi, Y.; Anpo, M.; Bahnemann, D. W. Understanding Tio2photocatalysis: Mechanisms and Materials. Chem. Rev. 2014, 114, 9919-9986.

(40) Abdellah, M.; Marschan, R.; Žídek, K.; Messing, M. E.; Abdelwahab, A.; Chábera, P.; Zheng, K.; Pullerits, T. Hole Trapping: The Critical Factor for Quantum Dot Sensitized Solar Cell Performance. J. Phys. Chem. C 2014, 118, 25802-25808.

(41) Nam, Y.; Li, L.; Lee, J. Y.; Prezhdo, O. V. Strong Influence of Oxygen Vacancy Location on Charge Carrier Losses in Reduced $\mathrm{TiO}_{2}$ Nanoparticles. J. Phys. Chem. Lett. 2019, 10, 2676-2683. 